\title{
Variability and polarization in the inner jet of 3C 395
}

\author{
L. Lara ${ }^{1}$, A. Alberdi ${ }^{1}$, and J. M. Marcaide ${ }^{2}$ \\ 1 Instituto de Astrofísica de Andalucía (CSIC), Apdo. 3004, 18080 Granada, Spain \\ 2 Departamento de Astronomía, Universitat de Valéncia, 46100 Burjassot, Spain \\ Received 10 November 2000 / Accepted 8 January 2001

\begin{abstract}
We present new results on the parsec-scale jet of the quasar 3C 395, derived from VLBI polarizationsensitive observations made in 1995.91 and 1998.50 at 8.4, 15.4 and $22.2 \mathrm{GHz}$. The observations show a complex one-sided jet extending up to 20 mas, with a projected magnetic field essentially aligned with the radio jet. The emission is strongly dominated, in total intensity and polarization, by the core and the inner jet region (of $\sim 3$ mas length). We have studied the details of this dominant region finding clear structural variations during this $\sim 2.5$ year period, in contrast with the apparent quietness of the jet structure inferred from lower resolution VLBI observations. We observe the ejection of a new component from the core and variations in the degree of polarization of the inner jet components. We estimate a high Faraday Rotation Measure close to the core, with a strong decrease along the inner jet.
\end{abstract}

Key words. galaxies: active - galaxies: individual: 3C 395 - galaxies: jets - radio continuum: galaxies techniques: interferometric

\section{Introduction}

The quasar 3C $395(1901+319 ; z=0.635)$ presents at milliarcsecond scales a radio structure which basically consists of two components (hereafter A and B), which are stationary with respect to each other and have a separation of $\sim 16$ mas, at a position angle of $118^{\circ}$. A third weak and extended component $(\mathrm{C})$ appears located between the two in maps made at frequencies lower than $15 \mathrm{GHz}$ (Lara et al. 1994, 1997). A flux density monitoring at 5.0, 8.0 and $14.5 \mathrm{GHz}$ made by the University of Michigan Radio Astronomy Observatory (UMRAO) shows that 3C 395 exhibits strong variability which presumably is the result of activity within component $\mathrm{A}$. In fact, high resolution space Very Long Baseline Interferometry (VLBI) observations resolve component A into a core-jet structure with bends. The existence of this complex structure might clarify the apparent contradiction between the lack of observed structural changes from lower resolution VLBI observations and the flux density variability (Lara et al. 1999). The stationary component $\mathrm{B}$ has been interpreted as the result of a local bend in the jet towards the observer, while component $\mathrm{C}$ seems more related to the emission of a complex underlying jet flow (see Lara et al. 1994, 1997, 1999). Polarimetric observations of 3C 395 have recently been reported by Taylor (2000), who presents, in the framework of a study of Faraday Rotation Measure (RM) in the inner jets of a sample of quasars, polarization sensitive VLBA ${ }^{1}$

Send offprint requests to: L. Lara, e-mail: lucas@iaa.csic.es

1 Very Long Baseline Array, operated by the National Radio Astronomy Observatory (NRAO). observations at frequencies between 4.6 and 15.2 GHz. Taylor finds a strong gradient in the RM of component A, with the highest value $\left(\sim+1200 \mathrm{rad} \mathrm{m}^{-2}\right)$ at the western end of this component.

It is now clear that the understanding of the activity and evolution of 3C 395 requires the study of the structure of component A at sub-milliarcsecond resolution. Accordingly, we present in this paper polarizationsensitive observations of the quasar 3C 395 made with the VLBA at 8.4, 15.4 and $22.2 \mathrm{GHz}$, with angular resolutions reaching 0.32 mas $\left(\simeq 1.24 \mathrm{pc}^{2}\right)$ at $22.2 \mathrm{GHz}$.

\section{Observations and data reduction}

We carried out VLBA observations of the quasar 3C 395 switching between frequencies at 8.4 and $15.4 \mathrm{GHz}$ in November 27th 1995 (epoch 1995.91) and at 15.4 and $22.2 \mathrm{GHz}$ in June 30th 1998 (epoch 1998.50). Left and right circular polarizations were recorded during both observing runs. The synthesized bandwidths per circular polarization were $16 \mathrm{MHz}$ and $32 \mathrm{MHz}$ in 1995 and 1998, respectively.

The correlation of the data was done in absentia by the staff of the VLBA correlator in Socorro (NM, USA). We used the NRAO AIPS ${ }^{3}$ package to correct for instrumental phase and delay offsets between the separate baseband

\footnotetext{
${ }^{2}$ We assume $H_{0}=100 \mathrm{Km} \mathrm{s}^{-1} \mathrm{Mpc}^{-1}$ and $q_{0}=0.5$ throughout.

3 Astronomical Image Processing System, developed and maintained by the NRAO.
} 
converters in each antenna, and to determine antennabased fringe corrections. The visibility amplitudes were calibrated using the system temperatures and gain information provided for each telescope. We estimate amplitude calibration errors to be smaller than $5 \%$ at all observing frequencies.

In both epochs, the determination of the feed responses to the polarized signal at each antenna was done using the feed solution algorithm of Leppänen et al. (1995), which calculates the so called D-terms (that is, the terms describing the "leakage" of the orthogonal polarization into each feed). Independent determinations of the instrumental polarization were done for each observed source, including 3C 395, obtaining a good agreement in the results at all frequencies and epochs. This allowed us to apply the corrections derived from a given source in the polarization mapping of that source. During the calibration, we assumed that the circularly polarized emission from all the sources was negligible, as suggested by the data. In 1995 , the number of known sources suitable for polarization calibration of VLBI observations at frequencies higher than $15 \mathrm{GHz}$ was very small. We took snapshots of the sources $1656+053,2145+067,3 \mathrm{C} 84$ and OQ 208, hoping to find a good calibrator of the absolute orientation of the electric field vector (electric vector position angle - EVPA) among them. The final EVPA was derived from the direct comparison of Very Large Array (VLA; close-in-time data were requested from the VLA public archive) and our VLBA polarization images of $1656+053$, which presents a compact structure at the angular resolutions provided by both instruments. From the VLA data we determined an $\mathrm{EVPA}=-15^{\circ}$ for this source. This orientation was consistent with that derived from the D-terms determined by Leppänen from a close-in-time VLBA experiment at 8.4 $\mathrm{GHz}$ (private communication). In 1998 the number of known polarization calibrators had increased considerably. We observed 3C 279, 1611+343 and 0420-014, all with known polarization properties, and we could obtain a consistent calibration for all of them. The absolute EVPA was derived from 0420-014, comparing our VLBA observations with the UMRAO database, and from the outer component in 3C 279 (Taylor \& Myers 2000). The EVPA were determined with an error that we estimate to be within $10^{\circ}$ at all epochs and frequencies.

Data imaging in total intensity was performed with the Difmap package (Shepherd et al. 1994). Maps of the Stokes parameters $Q$ and $U$ were made and combined in AIPS to finally obtain maps of the linearly polarized emission of 3C 395.

\section{Results}

Radio images of the compact structure of 3C 395 are shown in Fig. 1. They were obtained by applying natural weighting to the data. Total intensity maps are displayed with superimposed vectors representing the electric field.

\subsection{Total intensity}

At $8.4 \mathrm{GHz}$, we can identify the "classical" components A, B, and C (Fig. 1a), although A shows an elongated structure in the $\mathrm{S}-\mathrm{E}$ direction as an indication of its now-known complex composition. The jet can be traced continuously from $\mathrm{A}$ to $\mathrm{B}$, although we find no evidence of the radio jet beyond the latter component (Saikia et al. 1990; Lara et al. 1997, 1999). There is a sharp decrease in the intensity profile beyond component $\mathrm{A}$. The total flux density recovered in the VLBI map is $1.70 \pm 0.09 \mathrm{Jy}$.

The images at $15.4 \mathrm{GHz}$ from 1995 and 1998 (Figs. 1b-c) show only emission from components A and $\mathrm{B}$, while component $\mathrm{C}$ is resolved out and is too faint to be detected. The observations at this frequency, separated by almost three years, confirm the constant separation between components A and B. Moreover, the structure of B has remained essentially invariable during this period of time. On the other hand, A shows a bent core-jet structure with clear changes between the two epochs, probably resulting from a new ejected component (see also Fig. 3). The total flux density recovered in the VLBI maps at $15.4 \mathrm{GHz}$ is the same in both epochs, $1.12 \pm 0.06 \mathrm{Jy}$, despite the structural variations in component $\mathrm{A}$. Close-in-time space-VLBI observations at $4.85 \mathrm{GHz}$ (1 May 1998; Lara et al. 1999), which provide an angular resolution comparable to our $15.4 \mathrm{GHz}$ observations, show a similar bent core-jet structure.

At $22.2 \mathrm{GHz}$ (Fig. 1d), the detailed structure of component A is even more evident, consisting of an unresolved component at the western end of the brightness distribution (assumed to be the true core), a new component at a distance of 0.8 mas from the core at position angle $\mathrm{PA} \sim 110^{\circ}$ and a jet-like feature directed along $\mathrm{PA} \sim 130^{\circ}-140^{\circ}$. The flux density in the VLBI map is $0.94 \pm 0.05 \mathrm{Jy}$.

We have fitted simple elliptical Gaussian components to the visibility data using a least square algorithm within the Difmap package in order to obtain a quantitative description of the milliarcsecond structure of 3C 395 . Parameters describing the Gaussian components for each epoch and frequency are given in Table 1. Component A requires a rather complex model, three Gaussian components, to satisfactorily reproduce the $u v$-data: A1 stands for the core; A2 describes the new component which is clearly separated from the core in 1998.50; A3 describes the outer jet emission beyond component A2 (see Fig. 1d). Component B is described in terms of 2 Gaussian components to account for its compact and extended emission, respectively. Finally, component $\mathrm{C}$, only detected at $8.4 \mathrm{GHz}$, is rather weak and extended and it is represented in terms of a single and elongated Gaussian component. We also include in Table 1 a similar fit obtained at $4.85 \mathrm{GHz}$ in 1998.33 (Lara et al. 1999), but modified to describe $\mathrm{B}$ with two components, instead of the three used in the quoted publication.

If we combine the close-in-time data at epoch 1998 (4.85 GHz on 1998.33 and $15.4 / 22.2 \mathrm{GHz}$ on 1998.50 ) with 

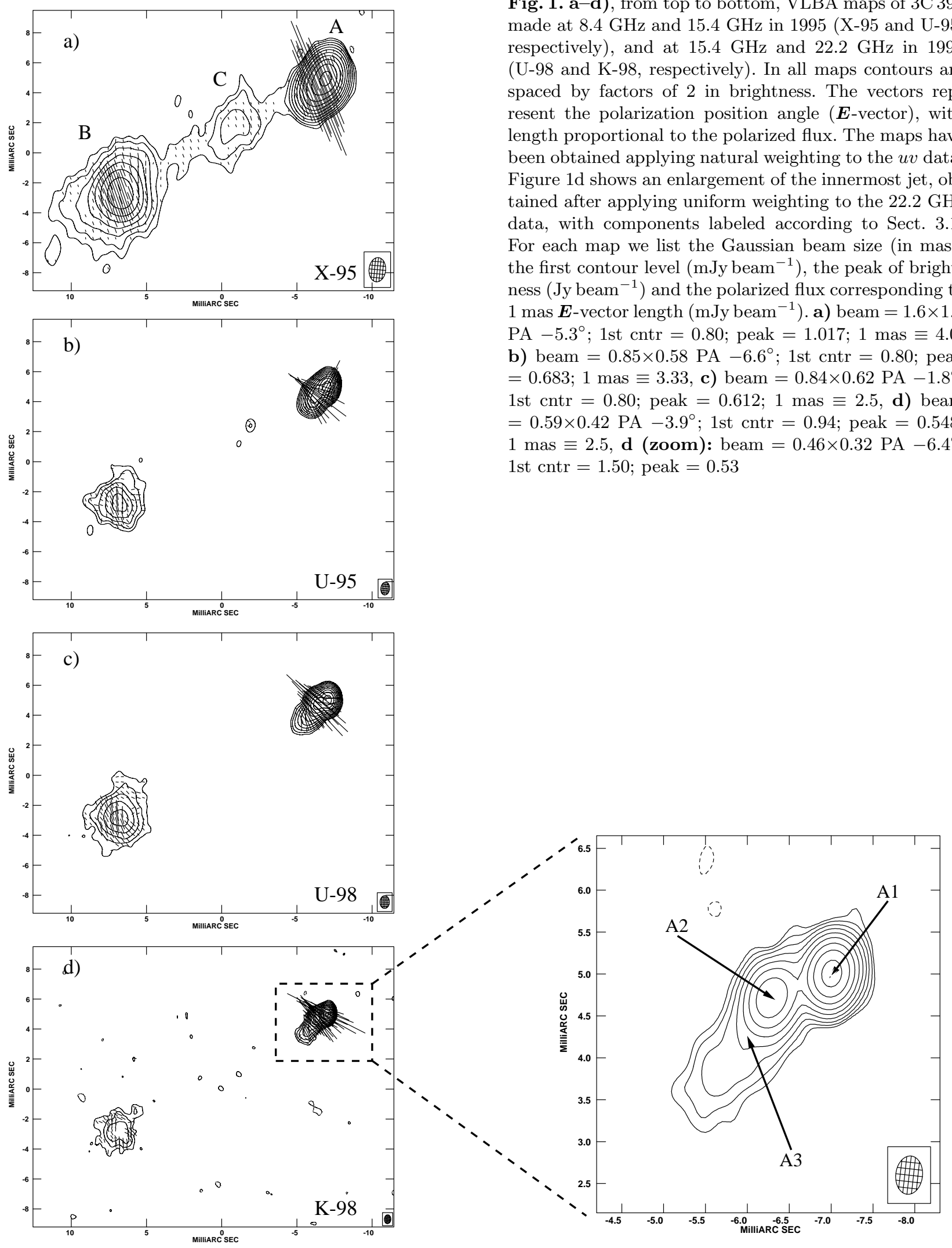

Fig. 1. a-d), from top to bottom, VLBA maps of 3C 395 made at $8.4 \mathrm{GHz}$ and $15.4 \mathrm{GHz}$ in 1995 (X-95 and U-95, respectively), and at $15.4 \mathrm{GHz}$ and $22.2 \mathrm{GHz}$ in 1998 (U-98 and K-98, respectively). In all maps contours are spaced by factors of 2 in brightness. The vectors represent the polarization position angle (E-vector), with length proportional to the polarized flux. The maps have been obtained applying natural weighting to the $u v$ data. Figure 1d shows an enlargement of the innermost jet, obtained after applying uniform weighting to the $22.2 \mathrm{GHz}$ data, with components labeled according to Sect. 3.1. For each map we list the Gaussian beam size (in mas), the first contour level $\left(\mathrm{mJy}\right.$ beam $\left.^{-1}\right)$, the peak of brightness $\left(\mathrm{Jy} \mathrm{beam}^{-1}\right)$ and the polarized flux corresponding to 1 mas $\boldsymbol{E}$-vector length $\left(\mathrm{mJy}_{\mathrm{beam}}{ }^{-1}\right)$. a) beam $=1.6 \times 1.1$ $\mathrm{PA}-5.3^{\circ} ; 1$ st $\mathrm{cntr}=0.80 ;$ peak $=1.017 ; 1$ mas $\equiv 4.0$, b) beam $=0.85 \times 0.58 \mathrm{PA}-6.6^{\circ} ; 1$ st $\mathrm{cntr}=0.80$; peak $=0.683 ; 1$ mas $\equiv 3.33$, c) beam $=0.84 \times 0.62 \mathrm{PA}-1.8^{\circ}$; 1 st $\mathrm{cntr}=0.80 ;$ peak $=0.612 ; 1$ mas $\equiv 2.5$, d) beam $=0.59 \times 0.42 \mathrm{PA}-3.9^{\circ} ; 1$ st $\mathrm{cntr}=0.94 ;$ peak $=0.548$; 1 mas $\equiv 2.5, \mathbf{d}($ zoom $):$ beam $=0.46 \times 0.32 \mathrm{PA}-6.4^{\circ}$; 1 st $\operatorname{cntr}=1.50 ;$ peak $=0.53$ 
Table 1. Gaussian components derived from the model fitting

\begin{tabular}{|c|c|c|c|c|c|c|c|}
\hline & $\mathrm{ID}^{a}$ & $\begin{array}{r}S^{b} \\
(\mathrm{mJy})\end{array}$ & $\begin{array}{r}D^{c} \\
(\mathrm{mas})\end{array}$ & $\begin{array}{c}\mathrm{PA}^{d} \\
\left({ }^{\circ}\right) \\
\end{array}$ & $\begin{array}{r}L^{e} \\
(\mathrm{mas})\end{array}$ & $\overline{r^{f}}$ & $\begin{array}{c}\Phi^{g} \\
\left({ }^{\circ}\right)\end{array}$ \\
\hline \multirow[t]{5}{*}{$\mathrm{A} 1$} & X95 & 741 & - & - & 0.22 & $?$ & 133 \\
\hline & U95 & 691 & - & - & 0.20 & 0.17 & 106 \\
\hline & U98 & 617 & - & - & 0.16 & 0.28 & 115 \\
\hline & K98 & 570 & - & - & 0.14 & $?$ & 107 \\
\hline & C98 & 124 & - & - & 0.59 & $?$ & 106 \\
\hline \multirow[t]{5}{*}{ A2 } & X95 & 353 & 0.56 & 113 & 0.63 & 0.12 & 134 \\
\hline & U95 & 179 & 0.61 & 115 & 0.56 & 0.30 & 132 \\
\hline & U98 & 314 & 0.76 & 112 & 0.32 & 0.49 & 131 \\
\hline & K98 & 211 & 0.77 & 111 & 0.26 & 0.49 & 129 \\
\hline & C98 & 629 & 0.66 & 109 & 0.30 & 0.74 & 132 \\
\hline \multirow[t]{5}{*}{ A3 } & X95 & 323 & 1.25 & 135 & 0.66 & 0.50 & 119 \\
\hline & U95 & 142 & 1.30 & 135 & 0.50 & 0.59 & 101 \\
\hline & U98 & 73 & 1.50 & 132 & 1.09 & 0.38 & 131 \\
\hline & K98 & 84 & 1.06 & 126 & 1.71 & 0.23 & 140 \\
\hline & C98 & 245 & 1.57 & 126 & 0.58 & 0.71 & 160 \\
\hline \multirow[t]{2}{*}{$\mathrm{C}$} & X95 & 25 & 6.97 & 119 & 4.60 & 0.44 & 127 \\
\hline & C98 & 48 & 7.60 & 118 & 8.70 & 0.22 & 118 \\
\hline \multirow[t]{5}{*}{ B1 } & X95 & 168 & 15.86 & 120 & 1.46 & 0.80 & 38 \\
\hline & U95 & 71 & 15.88 & 120 & 1.48 & 0.77 & 45 \\
\hline & U98 & 72 & 15.92 & 120 & 1.53 & 0.79 & 64 \\
\hline & K98 & 31 & 15.96 & 120 & 1.62 & 0.57 & 53 \\
\hline & C98 & 270 & 16.00 & 120 & 1.54 & 0.97 & 25 \\
\hline \multirow[t]{5}{*}{ B2 } & X95 & 82 & 16.15 & 118 & 4.47 & 0.55 & 138 \\
\hline & U95 & 30 & 15.81 & 117 & 3.55 & 0.40 & 137 \\
\hline & U98 & 28 & 15.81 & 118 & 3.95 & 0.46 & 150 \\
\hline & K98 & 34 & 15.78 & 119 & 2.63 & 0.47 & 137 \\
\hline & C98 & 79 & 16.83 & 117 & 6.18 & 0.40 & 137 \\
\hline
\end{tabular}

${ }^{a}$ Frequency and year of observation. C, X, U and $\mathrm{K}$ stand for 4.85, 8.4, 15.4 and $22.2 \mathrm{GHz}$, respectively.

${ }^{b}$ Flux density of the Gaussian component.

${ }^{c}$ Angular distance from the western-most component A1.

${ }^{d}$ Position angle with respect to A1, measured north through east.

${ }^{e}$ Length of the major axis of the Gaussian component.

${ }^{f}$ Ratio between the major and minor axis of the Gaussian component.

${ }^{g}$ Orientation of the major axis, defined in the same sense as the position angle.

A question-mark indicates that the parameter involved cannot be well constrained by our data.

the results shown in Table 1, we can derive the spectra for the different components of 3C 395 (Fig. 2). The spectral decomposition shows that the core peaks at a frequency between 5 and $15 \mathrm{GHz}$ in 1998. On the other hand, the other jet components (A2, B1) show a typical steep spectrum with spectral index $\alpha_{\mathrm{A} 2}=-0.68, \alpha_{\mathrm{B} 1}=-1.35$ (the spectral index $\alpha$ is defined so that the flux density $\left.S \propto \nu^{\alpha}\right)$. Considering the data at $8.4 / 15.4 \mathrm{GHz}$ on 1995.91, we can confirm the steep spectrum for A2 and B1, and a flat spectrum for the core $\left(\alpha_{\mathrm{A} 1}=-0.12\right)$, consistent with a shift of the turnover frequency towards higher frequencies at the later epoch. It is interesting to note that, in both epochs, the spectrum steepens with increasing core separation.

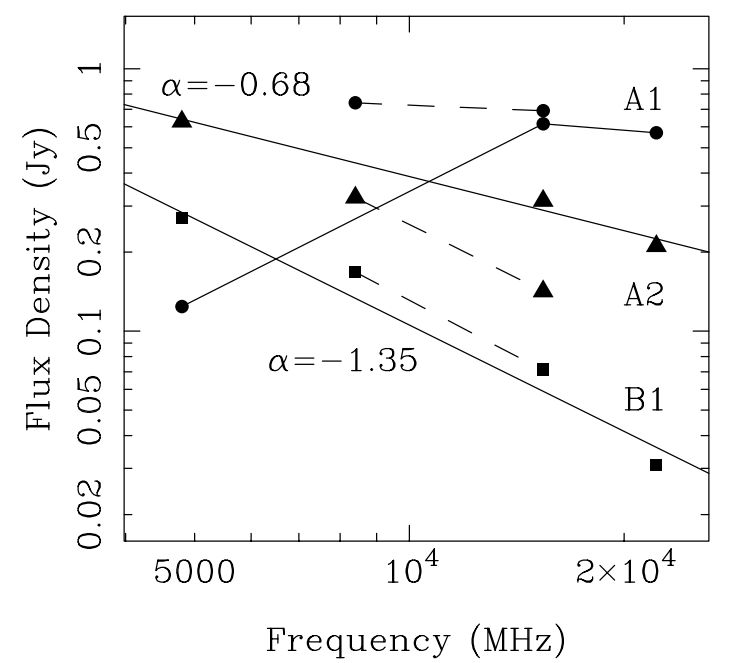

Fig. 2. Spectrum of components A1 (circles), A2 (triangles) and B1 (squares) of 3C 395 at epoch 1995 (dashed line) and epoch 1998 (solid line). See Table 1 for numerical values. The lines represent linear fits to the data of each component, excepting A1, for which the line helps to visualize the spectral dependence of its flux density. Spectral index values of B1 and A2 correspond to epoch 1998

The components A3 and B2 show also steep spectra in both epochs. We have not included them in Fig. 2 since they fit the extended emission of the inner jet and have different angular sizes in our model fitting, depending on the observing frequency.

\subsection{Polarized emission}

The polarized emission of 3C 395 comes predominantly from component A. The orientation of the magnetic field is essentially aligned with the radio jet, except at the compact core (A1), where we observe a strong dependence of the polarized intensity and of the magnetic field orientation with the frequency and epoch of observations. We further discuss this issue in the next section.

The polarized structure of component B is complex. Since this component is probably the result of the Doppler boosting of the radiation from a portion of a relativistic jet which is sharply directed towards the observer (Lara et al. 1994), the changes in the orientation of the magnetic vector in this region might reflect the change of orientation of the jet trajectory with respect to the line of sight. It is interesting to note that the configuration of the magnetic field suggests that the interaction of the jet with the surrounding medium is not significant, otherwise the magnetic field would be compressed and disposed perpendicularly to the jet direction. This result supports the idea that component $\mathrm{B}$ is a consequence of a geometry effect in the jet. Moreover, the fact that component $\mathrm{B}$ does not show any dependence of the magnetic field orientation and magnitude with time is consistent with its stationary character. 
Component $\mathrm{C}$ is only detected at $8.4 \mathrm{GHz}$ with polarized emission slightly above the noise level. However, we observe that the magnetic field tends to be parallel to the jet trajectory in this component. This fact, joined with its large size, does not lend support to the interpretation of $\mathrm{C}$ as a moving shocked component, favoring the hypothesis of $\mathrm{C}$ being mainly the result of the underlying jet emission.

\section{Discussion}

\subsection{Structural variability}

The quasar 3C 395, considered in the 1980's as being among the fast superluminal sources (Waak et al. 1985; Simon et al. 1988), showed, however, a stationary structure in observations made during the 90's (Lara et al. 1997), even though pronounced flux density variations are usually observed in this source (UMRAO database). Lara et al. (1999) found a large bend in the inner region of the jet, close to the core, and suggested that it could explain why previous Earth-based cm-VLBI observations did not detect the ejection of the new moving components expected from the flux density variability: a large bend in the inner jet would make it very difficult to correlate flux density variability within A with structural variations beyond this component because the effect of relativistic time-delay makes the time scales of these two events very different, and the decrease in the Doppler factor after the bend produces a large diminution in the flux density of possible moving components. As a step forward in the understanding of this source, the observations presented here show clear evidence of structural variability close to the core of 3C 395 between 1995 and 1998, in agreement with the previous scenario. In Fig. 3 we display the brightness distribution in total intensity and polarization of component A, as observed at $15.4 \mathrm{GHz}$ in 1995.91 and 1998.50 , respectively. Changes are evident, both in total intensity and polarization. The structural variability is consistent with a new component (labeled A2 in Table 1) which has been ejected from the core and is traveling along the jet. From the results of the model fitting at $15.4 \mathrm{GHz}$ we estimate an apparent velocity of $\beta_{\text {app }}=1.2 \pm 0.8 \mathrm{c}$ for this component, where the error is calculated assuming a conservative uncertainty in the position equal to one fifth of the beam size.

\subsection{Polarization in the inner jet region}

In Table 2 we display, for the different frequencies and epochs of observations, the total and polarized flux densities, the mean fractional polarizations and the mean EVPAs of the components of 3C 395. Total flux densities are taken from Table 1 while polarized flux densities were measured from the polarization images, defining a polygonal area containing all the component emission. We were not able to discriminate between the polarized emission from components $\mathrm{A} 2$ and $\mathrm{A} 3$, or $\mathrm{B} 1$ and $\mathrm{B} 2$, since they
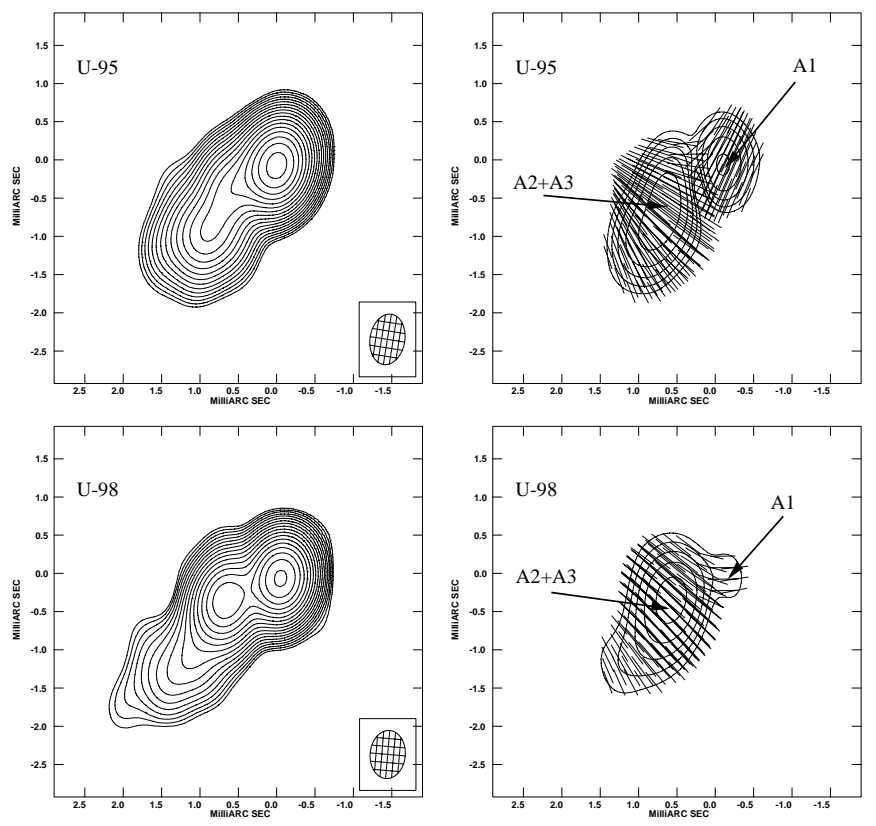

Fig. 3. Structural variations in total intensity (left) and polarization (right) in the inner structure of 3C 395, observed at $15.4 \mathrm{GHz}$ between 1995.91 and 1998.50. The contours are spaced by factors of $\sqrt{2}$ in all maps. Vectors represent the same as in Fig. 1. For each map we list the Gaussian beam size (in mas), the first contour level $\left(\mathrm{mJy}_{\mathrm{beam}}{ }^{-1}\right)$, the peak of brightness $\left(\mathrm{Jy}_{\mathrm{beam}}{ }^{-1}\right)$ and in polarization maps also the polarized flux corresponding to 1 mas $\boldsymbol{E}$-vector length $\left(\mathrm{mJy}_{\mathrm{beam}}{ }^{-1}\right)$. U-95 left: beam $=0.67 \times 0.45 \mathrm{PA}-9.0^{\circ} ; 1$ st $\mathrm{cntr}=1.5$; peak $=0.652$. U-95 right: beam $=0.94 \times 0.64 \mathrm{PA}-6.1^{\circ} ; 1$ st $\mathrm{cntr}$ $=3$; peak $=0.016 ; 1$ mas $\equiv 10$. U-98 left: beam $=0.63 \times 0.46$ $\mathrm{PA}-4.9^{\circ} ; 1$ st $\mathrm{cntr}=1.5 ;$ peak $=0.588 ; 1$ mas $\equiv 2.5 . \mathbf{U}-\mathbf{9 8}$ right: beam $=0.92 \times 0.70 \mathrm{PA}-1^{\circ} ; 1$ st $\mathrm{cntr}=3$; peak $=0.014$; 1 mas $\equiv 10$

present similar EVPA. Thus, we give values for the blending of these components.

The core component, A1, shows strong variability in the polarized structure at $15.4 \mathrm{GHz}$ (see Fig. 3). The polarized flux density of this component decreases from 11 to $3 \mathrm{mJy}$ between 1995.91 and 1998.50. Although A1 is weak in the latter epoch, we are confident that it is a real feature in our map, at a level above $5 \sigma$. The EVPA also rotates significantly from $158^{\circ}$ to $\sim 93^{\circ}$ in this time period.

Comparing the results at 15.4 and $22.2 \mathrm{GHz}$ from 1998 (Fig. 4), we find that component A1 presents a strongly inverted spectrum in polarization $(15 \mathrm{mJy}$ at $22.2 \mathrm{GHz}$ and $3 \mathrm{mJy}$ at $15.4 \mathrm{GHz}$ ). Since in 1998 the new component A2 was completely ejected from the core region, we suggest that i) the "true" core is almost unpolarized at frequencies lower than $15 \mathrm{GHz}$, as expected from synchrotron self-absorption near the core, and ii) the polarization variations observed between 1995 and 1998 at $15.4 \mathrm{GHz}$ in A1 are most plausibly due to the process of ejection of component A2 and to the resulting opacity changes in the jet. From the 1998 data, a RM of $\sim+2500 \mathrm{rad} \mathrm{m}^{-2}$ can be estimated in component A1, in agreement with Taylor (2000), which is consistent with the depolarization of the core 
region at low frequencies. We derive an intrinsic orientation of the electric vector of $35^{\circ}$, in agreement with the orientation of $\mathrm{A} 2+\mathrm{A} 3$, and therefore with a magnetic field oriented along the jet. The origin of the Faraday rotation is unclear to us. While it might have an origin internal to the jet, the effects produced by a possible external ionized screen in the core region cannot be disentangled. We note that the effect of bandwidth depolarization is negligible with the frequencies and bandwidths used in our observations, even with the high RM involved in the core of $3 \mathrm{C} 395$.

Component $\mathrm{A} 2+\mathrm{A} 3$ shows an EVPA which has remained essentially constant $\left(\sim 40^{\circ}\right)$ at $15.4 \mathrm{GHz}$ from 1995 to 1998 . On the other hand, the overall degree of polarization decreases from $\sim 8 \%$ to $\sim 6 \%$ in this time period. Moreover, the gradual rotation of the electric vector along component A2+A3 is also noticeable (see Fig. 3). This is probably related to the curvature present in the jet, as suggested by Lara et al. (1999). At $8.4 \mathrm{GHz}$ it is not possible to discern the different components within A in polarization. However, since the peak of the polarized emission is coincident with the position of component A2 and assuming that A1 is almost unpolarized at this frequency, we can ascribe the observed EVPA to A2+A3. Comparing with results from 1995 at $15.4 \mathrm{GHz}$ we estimate for this component a $\mathrm{RM}$ of $\sim-350 \mathrm{rad} \mathrm{m}^{-2}$, which is consistent with the small variation of the EVPA observed between 15.4 and $22.2 \mathrm{GHz}$ in 1998.

If we interpret the moving component A2 as a planar shock wave, then the compression of the shock enhances the component of the underlying magnetic field parallel to the shock front (transverse to the jet trajectory). This could explain the different field orientation of the core component A1 with respect to $\mathrm{A} 2+\mathrm{A} 3$ at $15.4 \mathrm{GHz}$ in 1995. However, since the orientation of the magnetic field

Table 2. Polarization in 3C 395

\begin{tabular}{crrrrr}
\hline Component & $\mathrm{ID}^{a}$ & $\begin{array}{r}\mathrm{S}_{\text {tot }}^{b} \\
(\mathrm{mJy})\end{array}$ & $\begin{array}{r}\mathrm{S}_{\text {pol }}^{c} \\
(\mathrm{mJy})\end{array}$ & $\begin{array}{r}p_{\mathrm{m}}^{d} \\
(\%)\end{array}$ & $\begin{array}{c}\chi^{e} \\
\left({ }^{\circ}\right)\end{array}$ \\
\hline $\mathrm{A} 1+\mathrm{A} 2+\mathrm{A} 3$ & $\mathrm{X} 95$ & 1417 & 49 & 3.5 & 22 \\
& & & & & \\
$\mathrm{~A} 1$ & $\mathrm{U} 95$ & 691 & 11 & 1.6 & 158 \\
$\mathrm{~A} 1$ & $\mathrm{U} 98$ & 617 & 3 & 0.5 & $\sim 93$ \\
$\mathrm{~A} 1$ & $\mathrm{~K} 98$ & 570 & 15 & 2.6 & 64 \\
& & & & & \\
$\mathrm{~A} 2+\mathrm{A} 3$ & $\mathrm{U} 95$ & 321 & 25 & 7.8 & 40 \\
$\mathrm{~A} 2+\mathrm{A} 3$ & $\mathrm{U} 98$ & 387 & 24 & 6.2 & 42 \\
$\mathrm{~A} 2+\mathrm{A} 3$ & $\mathrm{~K} 98$ & 295 & 19 & 6.4 & 45 \\
& & & & & \\
$\mathrm{~B} 1+\mathrm{B} 2$ & $\mathrm{X} 95$ & 248 & 27 & 10.9 & 19 \\
$\mathrm{~B} 1+\mathrm{B} 2$ & $\mathrm{U} 95$ & 101 & 10 & 9.9 & 21 \\
$\mathrm{~B} 1+\mathrm{B} 2$ & $\mathrm{U} 98$ & 100 & 11 & 11.0 & 22 \\
$\mathrm{~B} 1+\mathrm{B} 2$ & $\mathrm{~K} 98$ & 65 & 6 & 9.2 & $15 ?$ \\
\hline
\end{tabular}

\footnotetext{
${ }^{a}$ Frequency and year of observation: X, U and $\mathrm{K}$ stand for 8.4, 15.4 and $22.2 \mathrm{GHz}$, respectively.

${ }^{b}$ Total flux density of the component(s).

${ }^{c}$ Polarized flux density of the component(s).

${ }^{d}$ Mean fractional polarization.

e Mean EVPA of the component(s).
}
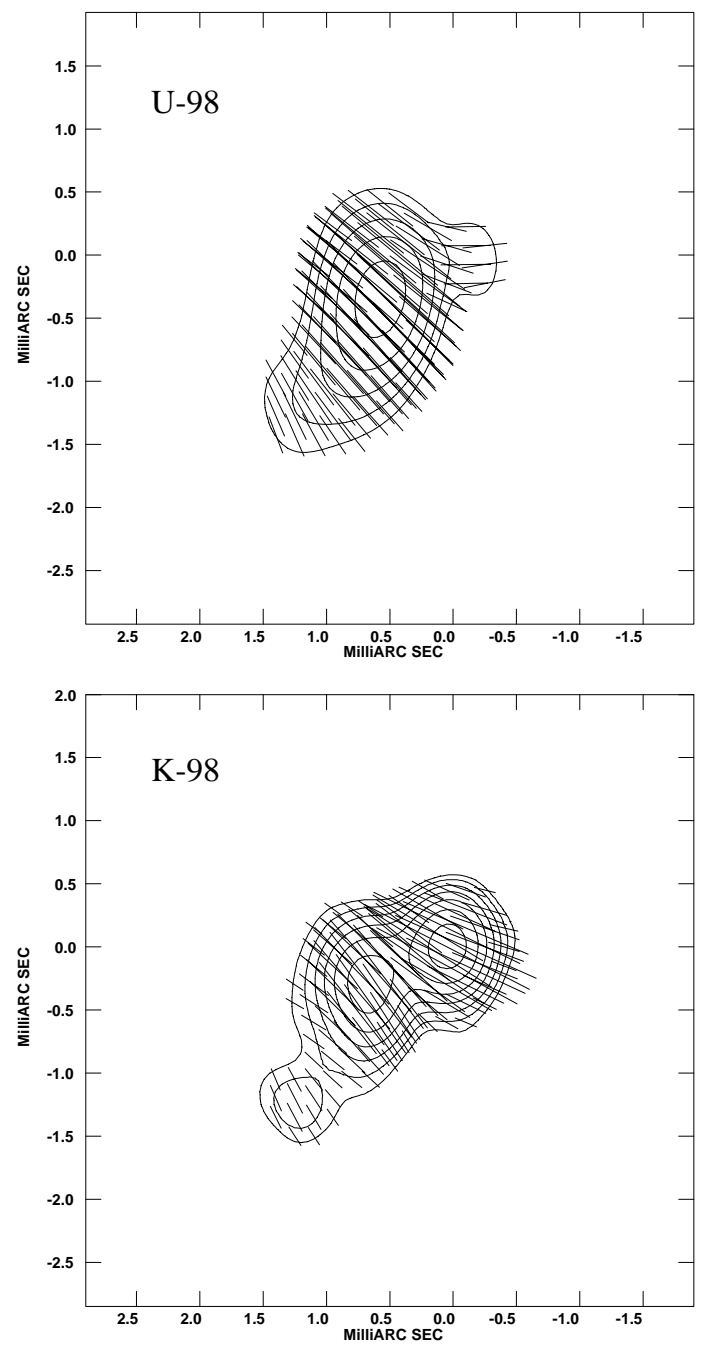

Fig. 4. Frequency dependent polarization variations in the very compact structure of 3C 395, observed at $15.4 \mathrm{GHz}$ and 22.2 GHz in 1998.50. The contours are spaced by factors of $\sqrt{2}$ in all maps. Vectors represent the same as in Fig. 1. For each map we list the Gaussian beam size (in mas), the first contour level $\left(\mathrm{mJy}_{\text {beam }}{ }^{-1}\right)$, the peak of brightness $\left(\mathrm{Jy} \mathrm{beam}^{-1}\right)$ and the polarized flux corresponding to 1 mas $\boldsymbol{E}$-vector length $\left(\mathrm{mJy}_{\text {beam }}{ }^{-1}\right)$. Top: beam $=0.92 \times 0.70 \mathrm{PA}-1^{\circ} ; 1$ st $\mathrm{cntr}=$ 3 ; peak $=0.014 ; 1$ mas $\equiv 10$. Bottom: beam $=0.67 \times 0.51 \mathrm{PA}$ $-2.9^{\circ} ; 1$ st $\operatorname{cntr}=1.5 ;$ peak $=0.015 ; 1$ mas $\equiv 10$

in 1998 is along the jet (once corrected of Faraday rotation), we should conclude that the enhancement of the field produced by the shock wave is not strong enough to dominate over the underlying parallel field component. This is consistent with the slight decrease in the degree of polarization at $15.4 \mathrm{GHz}$ observed between 1995 and 1998, and in agreement with the general tendency in quasars to have a net magnetic field orientation parallel to the jet (Cawthorne et al. 1993; Wardle 1998).

Finally, we note the presence of a strong gradient in the RM along the inner jet of 3C 395, in agreement with Taylor (2000). Strong gradients in relativistic jets have also been found in other compact sources, such as OJ 287 (Gabuzda \& Gómez 2000) and BL-Lac (Reynolds \& Cawthorne 2000). 


\subsection{Polarization in component $B$}

Component $\mathrm{B} 1+\mathrm{B} 2$ has a degree of polarization similar for all frequencies and observing epochs, which indicates that Faraday rotation is not significant in this region of 3C 395. We note that Taylor (2000) finds a moderate RM of $68 \pm 40 \mathrm{rad} \mathrm{m}^{-2}$ in component $\mathrm{B}$, which is fully consistent with our results. Moreover, the observed EVPA implies that the magnetic field is oriented along the jet. As previously mentioned (Sect. 3.2), the configuration of the magnetic field suggests that this component is not the result of a possible interaction of the jet with the external medium, and argues in favor of a change in the jet geometry.

\section{Conclusions}

We have made VLBA observations of the quasar 3C 395 at 8.4 and 15.4 in 1995.91, and at 15.4 and 22.2 in 1998.50, and have obtained detailed maps of its parsec-scale jet, in total intensity and in polarization. The one-sided jet is complex, showing the previously reported gross structure of three components, $\mathrm{A}, \mathrm{B}$ and $\mathrm{C}$, but with remarkable sub-structure when observed at sub-milliarcsecond angular resolution. The emission of 3C 395 is strongly dominated by component $\mathrm{A}$. The new data confirm the stationary character of component B, also in polarized emission. Component $\mathrm{C}$ appears very elongated at $8.4 \mathrm{GHz}$, being more likely the result of the underlying jet emission. It is resolved out at higher frequencies.

We have studied the sub-structure within component $\mathrm{A}$, i.e. the core and the inner jet region (of $\sim 3$ mas length), which is crucial to understand the relation between flux density and structural variability in 3C 395. We have found clear structural variations in the inner jet, with a new component ejected from the core. In polarization, we have observed also important time and frequency dependent variations of the inner jet structure, most possibly associated with the ejection of a new component from the core. The variability of this region of 3C 395 contrasts with the apparent stability inferred from lower angular resolution observations, which could only determine that total flux density variability was due to activity within component A.

The observations show that the jet of 3C 395 has a projected magnetic field essentially aligned with the direction of the jet, indicating that this parallel field is stronger than the field compression exerted by the shock wave associated with the new ejected component. The underlying parallel magnetic field is strong enough to be detected at a distance of 16 mas $(\sim 60 \mathrm{pc})$ from the core. We find good agreement when comparing the degree of polarization and the electric vector position angle obtained from our VLBA data (Table 2) and from close-in-time single dish data (database of the University of Michigan Radio Astronomy Observatory: EVPA $33^{\circ}$ and polarization degree of $3 \%$ at $15 \mathrm{GHz}$ in epoch 1998.37), indicating that the polarization of the source as a whole is mainly asso- ciated with the compact radio source and, in particular, components A and B.

Finally, we estimate a high Faraday RM, $\sim+2500 \mathrm{rad} \mathrm{m}^{-2}$, close to the core region, and a strong gradient along the inner jet, reaching $\mathrm{RM}$ $\sim-350 \mathrm{rad} \mathrm{m}^{-2}$ at a distance of only $2-3$ mas from the core. These values are in agreement with the results derived by Taylor (2000).

Follow up observations of moving components such as A2 in the inner jet of 3C 395 at high angular resolution, by means of high frequency and/or space-VLBI observations, would eventually allow us to trace the jet curvature close to the core before those components fade away below the noise level as they reach the sharp cut in the emission profile beyond component A. We guess that only very strong components would be detected in the region between components A and B, probably similar to the one observed during the 1980's (Waak et al. 1985).

Acknowledgements. We thank Kari Leppänen for his invaluable help during the 1995 data reduction process and the referee for helpful and constructive comments to the paper. A. A. acknowledges the European Commission's TMR Programme, Access to Large Scale Facilities, under contract No. ERBFMGECT950012 for providing financial support to visit the Joint Institute for VLBI in Europe (JIVE) for data reduction. This research is supported in part by the Spanish DGES Grant (PB97-1164) and has made use of data from the University of Michigan Radio Astronomy Observatory which is supported by funds from the University of Michigan. The National Radio Astronomy Observatory is a facility of the National Science Foundation operated under cooperative agreement by Associated Universities, Inc.

\section{References}

Cawthorne, T. V., Wardle, J. F. C., Roberts, D. H., \& Gabuzda, D. C. 1993, ApJ, 416, 519

Gabuzda, D., \& Gómez, J. L. 2000, MNRAS, in press

Lara, L., Alberdi, A., Marcaide, J. M., \& Muxlow, T. W. B. 1994, A\&A, 285, 393

Lara, L., Muxlow, T. W. B., Alberdi, A., et al. 1997, A\&A, 319,405

Lara, L., Alberdi, A., Marcaide, J. M., \& Muxlow, T. W. B. 1999, A\&A, 352, 443

Leppänen, K., Zensus, J. A., \& Diamond, P. J. 1995, AJ, 110, 2479

Reynolds, C., \& Cawthorne, T. V. 2000, MNRAS, submitted

Saikia, D. J., Muxlow, T. W. B., \& Junor, W. 1990, MNRAS, 245,503

Shepherd, M. C., Pearson, T. J., \& Taylor, G. B. 1994, BAAS, 26,987

Simon, R. S., Hall, J., Johnston, K. J., et al. 1988, ApJ, 326, L5

Taylor, G. B. 2000, ApJ, 533, 95

Taylor, G. B., \& Myers, S. T. 2000, VLBA Scientific Memorandum, No. 26

Waak, J. A., Spencer, J. H., Johnston, K. J., \& Simon, R. S. 1985, AJ, 90(10), 1989

Wardle, J. F. C. 1998, in IAU Colloq. 164: Radio Emission from Galactic and Extragalactic Compact Sources, ed. J. A. Zensus, G. B. Taylor, \& J. M. Wrobel, ASP Conf. Ser., 144,97 